\title{
Clinical, electrophysiological findings and evaluation of prognosis of patients with Guillain-Barré syndrome
}

\author{
Betül Kılıç, Serdal Güngör, Bilge Özgör \\ Department of Pediatric Neurology, Inönü University Faculty of Medicine, Malatya, Turkey. E-mail: betulklc82@gmail.com \\ Received: 22nd May 2018, Revised: 27th June 2018, Accepted: 6th July 2018
}

SUMMARY: Kılı̨̧ B, Güngör S, Özgör B. Clinical, electrophysiological findings and evaluation of prognosis of patients with Guillain-Barré syndrome. Turk J Pediatr 2019; 61: 200-208.

Guillain-Barré syndrome (GBS) is an acute inflammatory polyneuropathy characterized by frequent rapid progressive, ascending, symmetric weakness and areflexia. We aimed to evaluate the etiology, clinical and electrophysiological findings with treatment and prognosis of the patients with GBS in our clinic. Patients who were diagnosed with GBS in our clinic between 2009 and 2017 were evaluated retrospectively. The study included 20 female and 25 male patients. The most frequent symptom was the absence of walking (95.5\%). All of the patients had muscle weakness on examination; in addition to that hyperesthesia $(31 \%)$, autonomic symptoms (13.3\%), sensory loss $(11.1 \%)$, ataxia $(11.1 \%)$, bilateral facial nerve palsy $(6.6 \%)$, oculomotor nerve palsy $(2.2 \%)$, and multiple cranial nerve involvement $(2.2 \%)$ were the other detected findings. Ventilation support was required in 6 cases $(13.3 \%)$. Acute motor axonal neuropathy (AMAN) was found in 20 patients $(44.5 \%)$, acute inflammatory demyelinating polyradiculoneuropathy (AIDP) was found in 24 patients $(53.3 \%)$, and acute motor and sensory axonal neuropathy (AMSAN) was only present in 1 patient $(2.2 \%)$. Intravenous immunoglobulin (IVIG) was administered to 33 of the patients $(73.3 \%)$. The mean hospital stay was $8.4 \pm 3.5$ (2-17 days), and the relationship between the duration of hospital stay and the treatment given was statistically significant $(p=0.001)$. Complete remission was observed in 37 patients $(82.3 \%)$ and the remaining 5 children $(11.1 \%)$ experienced incomplete recovery. Three patients $(6.7 \%)$ died of treatment-resistant hypotension, arrhythmia and severe pulmonary infection. The short duration of neurological deficit following infection, clinical stage of application, need for mechanical ventilation, dysautonomia, cranial nerve involvement, and current subtype were the negative prognostic factors. Although GBS is a self-limiting disease, early diagnosis and treatment are very important to reduce hospital stay with morbidity and mortality. Patients expected to be at high risk should be monitored closely.

Key words: Guillain-Barré syndrome, childhood, electrophysiology, intravenous immunoglobulin, prognosis.

Guillain-Barré Syndrome (GBS) is an acute, monophasic immune-mediated polyradiculoneuropathy caused by acute demyelination or axonal damage in the spinal roots and peripheral nerve. It is characterized by common sensory, motor and autonomic symptoms, usually accompanied by progressive flask paralysis and deep tendon reflex diminution. ${ }^{1}$ On average, $40-70 \%$ of GBS patients develop the disease approximately 1-3 weeks after acute gastroenteritis or respiratory tract infection. Rare cases occur in the course of surgery or the course of other diseases. ${ }^{2}$ GBS is thought to be an autoimmune disease resulting in the production of antibodies against antigenic proteins of peripheral nerves that result in $\mathrm{T}$ cell activation. Production of antibodies can be stimulated by infectious agents such as Ebstein-Barr virus, Cytomegalovirus, Mycoplasma pneumoniae and Campylobacter jejuni, vaccines or 
surgical interventions. As well as antibodies targeting myelin proteins, in some situations, axonal structures are the primary target of immune-modulated damage. Therefore, although GBS is known as acute inflammatory demyelinating polyneuropathy, in some clinical variants the primary pathological process is axonal damage. ${ }^{1}$

Several clinical subtypes of the syndrome have been described. 3,4 GBS has been reclassified in recent years according to clinical and electrophysiological properties. ${ }^{3,5}$ According to that, the large spectrum varies from classic acute inflammatory demyelinating polyneuropathy (AIDP) and the axonal forms; which include acute motor and sensory axonal neuropathy (AMSAN) and acute motor axonal neuropathy (AMAN) to several atypical clinical types (i.e., Miller Fisher syndrome, Bickerstaff brainstem encephalitis, pharyngo-cervicalbrachial variant, polyneuritiscranialis, acute sensory neuropathy and pandysautonomia). ${ }^{3,5}$ GBS usually has a good prognosis. $90 \%$ of patients recover completely or with mild neurological deficit within one year. ${ }^{6}$ In some studies, axonal involvement and duration of mechanical ventilation are shown to be predictors of poor prognosis in GBS. 7,8

Our aim in this study was to evaluate patients diagnosed with GBS in our clinic together with laboratory and electrodiagnostic findings concerning treatment and prognostic factors.

\section{Material and Methods}

In this study, etiology, clinical and electrophysiological prediction of the outcome, treatment, and findings were evaluated retrospectively in children who were diagnosed with GBS in our clinic between 2009-2016. Healthy children under 18 years of age who were diagnosed with GBS were included in the study. Patients with acute neuropathy causing chronic inflammatory demyelinating polyneuropathy (CIDP), patients with comorbid diseases and underlying diseases such as an autoimmune disease, neurological deficit or progressive disorder in history were excluded.

Data was collected for the study such as demographic data's (age and gender), previous infections (especially upper respiratory tract infection and gastrointestinal system infections), amount of time between the onset of the disease and admission, clinical features, cranial nerve involvement, need for mechanical ventilation, cerebrospinal fluid (CSF) protein and cell examination, electrophysiological data, type and results of the treatment and complications of the performed procedures, length of hospital stay and prognosis.

Serological tests for Cytomegalovirus (CMV), Herpes simplex virus (HSV), Epstein-Barr virus (EBV), Varicella zoster (VZV), Mycoplasma pneumoniae, Hepatitis $B$ and $C$, and Haemophilus influenzae were performed. Stool samples were obtained for Campylobacter jejuni isolation. Serologic test for Campylobacter jejuni could not be performed for financial reasons. CSF examination was performed in the first week of hospitalization in all patients. Only in one patient who had cranial nerve involvement, an enzyme-linked immunosorbent assay was used to measure serum IgG antibodies that were produced against gangliosides GM1, GM1b, GD1a, GalNAc-GD1a, GD1b, GT1a, and GQ1b. These tests were not performed in all patients since they were expensive and their impact on the diagnosis or management was limited.

Plasma exchange treatment was considered with immunoadsorption technique over 4 to 6 cycles on a daily or alternate day basis. Intravenous immunoglobulin (IVIG) was administered as $0.4 \mathrm{~g} / \mathrm{kg}$ daily for five consecutive days.

The electrophysiological examination was performed in the first week of hospitalization in all patients. Also, needle EMG examination was performed. At least one motor and one sensory nerve were tested on the upper and lower limbs. Sensory nerve studies were performed on the median and sural nerves. $F$ response of all the extremities was recorded. Additionally, routine motor conduction studies were performed on the median, ulnar and tibial nerves using conventional procedures. The amplitude of the negative phase was measured for compound muscle action potentials and sensory nerve action potentials. In our study, the patients were classified into AIDP or AMAN based on the existing electrodiagnostic criteria. ${ }^{9}$ AMSAN was defined as the presence of AMAN pattern in motor nerve studies with sensory nerve action potential amplitude reduction more than $50 \%$ of the normal in two or more sensory nerves. Patients with a significant decrease or loss of deep tendon reflexes with 
acute progressive muscle weakness and typical electrophysiological findings and no apparent sensory level were included in the study.

Functional outcomes were listed according to GBS disability score and MRC (the Medical Research Council) total score. GBS disability scores were as following ${ }^{10}$; 0 : healthy state score, 1: minor symptoms and capable of running, 2: able to walk $10 \mathrm{~m}$ or more without assistance but unable to run; 3 : able to walk 10 $\mathrm{m}$ across an open space with help, 4: bedridden or chair bound, 5: requiring assisted ventilation for at least part of the day, 6: dead.

The Medical Research Council (MRC) total score includes the scores of six different muscle groups; shoulder abduction on both sides, elbow fibers, ankle extensors, hip joints, knee extensors and foot dorsiflexors, scores range between 60 (normal) to 0 (quadriplegia). ${ }^{10}$ All patients' GBS disability scores and MRC scores at the time of hospitalization and discharge were evaluated. In our outpatient clinic, GBS disability scores were recorded for the six months of follow-up.

For the statistical analysis, SPSS for Windows version 20.0 program was used. The findings and percentages were presented for the descriptive statistics. As a statistical method, parametric tests were used for comparison, and nonparametric tests were used for comparison of abnormal distribution data. Spearman test was used for correlation analysis. Values below $\mathrm{p}<0.05$ were considered statistically significant.

\section{Results}

The study included 45 children, 25 males and 20 females with GBS. In the study, male/female ratio was found to be 1.25 . The average age was $6.7 \pm 3.9$ (range: $1.5-15$ years). There were $32(71.1 \%)$ cases of infection. Thirteen patients $(26.6 \%)$ had no infection observed up to 4 weeks before the onset of GBS symptoms. The mean time from onset of disease to nadir in 32 patients was 7.28 days (range: 1-20 days). Of these antecedent illnesses, respiratory tract infection was found in 22 cases $(48.8 \%)$, diarrhea in eight cases (17.7\%), hepatitis A in one child $(2.2 \%)$, and chickenpox infection in one child (2.2\%). Also, there was one case of immunization with influenza vaccine one month before the illness. Respiratory tract infection was common in patients with the AIDP subtype while gastroenteritis was common in patients with AMAN; correlations were statistically significant $(p=0.03)$. Bacterial isolation could not be achieved in the stool samples for Campylobacter jejuni. The demographic characteristics of the patients and the predisposing disease factors are given in Table I.

When the development of GBS by season is examined, twenty patients were found to have GBS in the spring (March to May), 11 in summer (June to August), seven in autumn (September to November) and seven in winter (December to February).

The most frequent symptom was the inability to walk (95.5\%). The most common finding on physical examination was muscle weakness, and in only one case there was the onset of upper extremity weakness (Table II). Other examinations included hyperesthesia (14/45; $31.1 \%)$, autonomic symptoms $(6 / 45 ; 13.3 \%)$, sensory loss $(5 / 45 ; 11.1 \%)$, ataxia $(5 / 45$; $11.1 \%)$, bilateral facial nerve palsy $(4 / 45$; $8.8 \%)$, bilateral ptosis $(1 / 45 ; 2.2 \%)$, and multiple cranial nerve involvement $(1 / 45$; $2.2 \%)$. One patient who had multiple cranial nerve involvement showed bilateral cranial nerve III, IV, V, VI, VII, VIII, IX, X, $\mathrm{XI}$, and XII involvements, but no signs of cerebellar involvement, including ataxia or other symptoms, show signs of autonomic or sphincter dysfunction. During the hospital stay transient hypertension, hypotension, tachycardia was noted as autonomic symptoms in six cases $(13.3 \%)$. Six $(13.3 \%)$ children required assisted ventilation due to respiratory insufficiency. Functional outcomes according to GBS disability score at nadir were stage 2 in 16 patients, stage 3 in 24 patients, and stage 4 in five patients. Examination findings are given in Table II.

According to the cerebrospinal fluid analysis, the mean CSF protein was $80.6 \pm 58.5 \mathrm{mg} /$ dl (range: $15-255 \mathrm{mg} / \mathrm{dl}$ ), with a total cell count of 0-3 cells $/ \mathrm{mm}^{3}$. In the CSF amination, albuminocytological dissociation (i.e., an increased protein concentration without pleocytosis) occurred in 36 patients $(80 \%)$. We studied only one patient's serum antiganglioside antibody who had multiple cranial nerve involvement and found IgG reactivity against GQ1b. 
Table I. Demographic Characteristics of Patients and Predisposing Infectious Agents.

\begin{tabular}{lc}
\hline Characteristics & N (\%) \\
\hline Gender & $25(55.6)$ \\
Male & $20(44.4)$ \\
Female & \\
Age & $7(15.5)$ \\
$\quad<3$ years & $22(48.8)$ \\
$3-6$ years & $7(15.5)$ \\
$7-10$ years & $9(20.0)$ \\
$>10$ years & \\
Predisposing infectious agents & $3(6.6)$ \\
Cytomegalovirus & $2(4.4)$ \\
Mycoplasma pneumoniae & $2(4.4)$ \\
Herpes simplex virus & $1(2.2)$ \\
Measles & $1(2.2)$ \\
Epstein-Barr virus & $1(2.2)$ \\
Human rhinovirus & $1(2.2)$ \\
Parvovirus & $1(2.2)$ \\
Hepatitis A & $1(2.2)$ \\
Brucellosis & $1(2.2)$ \\
Pseudomonas aeruginosa & $31(68.8)$ \\
Microorganisms not shown &
\end{tabular}

When classified according to electroneuromyography (ENMG), the number of acute inflammatory demyelinating polyradiculoneuropathy (AIDP) cases also called the classical form (24/45; $53.3 \%$ ) were found to be similar with the number of acute motor axonal neuropathy (AMAN) cases $(20 / 45 ; 44.5 \%)$, and acute motor and sensory axonal neuropathy (AMSAN) cases $(1 / 45 ; 2.2 \%)$ (Table II). There was no significant difference between AIDP and AMAN / AMSAN regarding ENMG examination time $(\mathrm{p}=0.152)$. All cases with cranial nerve involvement, which were most notable according to clinical features, were of the AMAN type and required mechanical ventilation.

Duration of the onset of the symptoms after the infection was shorter in the axonal form (AMAN and AMSAN) compared to the demyelinating form (AIDP), however; this finding was statistically not significant $(\mathrm{p}=0.067)$.

Functional outcomes according to GBS disability score at nadir was lower in AMAN than AIDP (3.0 vs. 2.4, $\mathrm{p}=0.001$ ); also the nadir MRC was lower in AMAN than AIDP $(35.2 \pm 14.0$ vs. $44.7 \pm 75.0, p=0.014)$ indicating that motor impairment was more severe in AMAN.
Table II. Clinical Characteristics, Electrophysiological Findings, Treatment and Prognosis of Patients.

\begin{tabular}{lc}
\hline & N (\%) \\
\hline Clinical & $45(100.0)$ \\
Muscle weakness & $14(31.1)$ \\
Hyperestesia & $6(13.3)$ \\
Autonomic symptoms & $5(11.1)$ \\
Ataxia & $5(11.1)$ \\
Sensory loss & $4(8.8)$ \\
Bilateral facial nerve paralysis & $3(6.6)$ \\
Shortness of breath & $1(2.2)$ \\
Multiple cranial nerve involvement & \\
GBS disability score at nadir & $16(35.5)$ \\
2 & $24(53.3)$ \\
3 & $5(11.1)$ \\
4 & \\
MRC sum score at nadir & $5(11.1)$ \\
$60-51$ & $20(44.4)$ \\
$50-41$ & $13(28.8)$ \\
$40-31$ & $3(6.7)$ \\
$30-21$ & $4(8.8)$ \\
$20-0$ &
\end{tabular}

Electrophysiological findings

$\begin{array}{lc}\text { AIDP } & 24(53.3) \\ \text { AMAN } & 19(42.2) \\ \text { AMSAN } & 2(4.4)\end{array}$

Treatment

IVIG

$33(73.3)$

IVIG with plasmapheresis

$9(20.0)$

Plasmapheresis

$3(6.6)$

Prognosis

Full recovery

Incomplete recovery

AIDP: acute inflammatory demyelinating polyneuropathy, AMAN: acute motor axonal neuropathy, AMSAN: acute motor-sensory neuropathy, GBS: Guillain-Barré syndrome, IVIG: Intravenous immunoglobulin, MRC: The Medical Research Council

In our study we performed spinal cord imaging on 33 patients with gadolinium magnetic resonance, we found contrast material enhancement on cauda equina level in 19 patients. We obtained negative findings in 14 patients. There was no significant difference between axonal and demyelinating groups $(\mathrm{p}=0.259)$.

IVIG was administered to 33 (73.3\%) of the patients, nine patients received both plasma 
exchange and IVIG (20\%), and plasma exchange was administered to three $(6.6 \%)$ of the patients. Plasma exchange consisted mainly of AMAN, AMSAN cases with GBS disability scores of 4 and 5 , and also some patients were in need of mechanical ventilation in the first week. In the early period, physiotherapy program started with passive exercises. There was no statistically significant difference between the treatment groups in terms of complications in our study. The mean hospital stay was 9.4 4.1 days (range: 5-17 days) which was significantly longer in patients who were treated with combined plasma and IVIG $(p=0.001)$. During the first six months, complete recovery was observed in $37(82.2 \%)$ patients, and the remaining five $(11.1 \%)$ patients experienced incomplete recovery. These cases were mostly AMAN subtype (4/5). Three patients $(6.7 \%)$ died due to pulmonary infection and treatment-resistant cardiovascular complication. Axonal involvement was present in all of these cases, and bilateral facial paralysis was present only in one patient.

GBS disability score at six months was stage 0 in 33 patients, stage 1 in 3 patients, stage 2 in 4 patients, and stage 3 in 2 patients. When we compared GBS disability scores we found that higher scores in the onset of disease, also meant higher scores in the 6th month $(\mathrm{p}<0.001$ [95\% CI 1.5-2.2]). No association was found between GBS disability score at 6 th month and gender $(p=0.37)$, age $(p=0.91)$, cranial nerve involvement $(p=0.45)$, sensory deficits $(\mathrm{p}=0.64)$, having previous infections $(\mathrm{p}=0.89)$. In multivariate analysis, as GBS disability score at 6 th month, GBS disability score at nadir $(\mathrm{p}<0.001 ; 95 \%$ CI 1.4-2.2), AMAN subtype, $(\mathrm{p}=0.02 ; 95 \% \mathrm{CI} 0.1-1.1)$, the mean time from onset of the disease to nadir $(\mathrm{p}=0.01 ; 95 \% \mathrm{CI}$ 5.3-9.1), mechanical ventilation requirement $(p=0.0195 \%$ CI 1.1-1.3), and presence of autonomic manifestation $(\mathrm{p}<0.001 ; 95 \% \mathrm{CI}$ 0.1-1.1) were found as poor prognostic factors.

\section{Discussion}

GBS is one of the most important reasons for acute flask paralysis. The frequency of the disease was found to be $0.25-1.5 / 100.000$ under the age of 16 in community-based studies. ${ }^{11}$ Although some studies have reported that there is no gender difference in GBS, it is reported that the incidence is higher in males which is similar with our study $(\mathrm{M} / \mathrm{F}=1.25) .{ }^{12,13} \mathrm{GBS}$ can appear at any age. It is most common between the ages of 4-9.14 In our study, the mean age was $6.7 \pm 3.9$ years (1.5-15 years). The cases were mostly between 3-6 years old.

Although GBS was thought to be sporadic without seasonal activity, similar to previous studies, in our study it was found that GBS was more likely to appear in the spring (44.4\%), and in the summer (24.4\%),7,15,16

Previous studies have reported a preliminary infectious rate of $40-70 \% .{ }^{17}$ In our study, predominant infection was present in $71.1 \%$ of the patients, mostly seen were respiratory tract infection and gastroenteritis. There are also studies reporting that there may be preliminary facts for GBS in vaccination, surgical procedures, and stress events. ${ }^{18}$ In studies conducted there is a hypothesis offering that the vaccine may be associated with GBS because of possible effects on the immune system. However, it has been emphasized that the benefits of vaccines should not be underestimated in the prevention and reduction of morbidity and mortality. ${ }^{19}$ In our study, one of our cases had a vaccine story approximately a month before the onset of the symptoms.

Affected children often have weakness, fatigue, with difficulty to start walking or stair climbing, typically starting from the lower extremities and advancing towards the upper extremities in days to weeks. In some of the cases (15-20\%) weakness is primarily proximal. ${ }^{20}$ In our cases, the most frequent symptom was the inability to walk (95.5\%) and weakness (100\%). Only one patient had an upper extremities onset weakness, and the others had onset lower extremities.

Physical examination usually reveals symmetrical weakness and loss of deep tendon reflexes which progress upwards starting from the lower extremities. In childhood, GBS cranial nerve involvement is more frequent than in adults. Sensory loss is rarely seen but can be detected in $40 \%$ of the cases with a detailed physical examination. ${ }^{12}$ Autonomic dysfunctions such as irregular blood pressure, sinus tachycardia, pupillary anomalies and sweating disorders are seen in $12.5-25 \%$ of children with GBS. ${ }^{20}$ Although extremely rare, GBS initially can present as isolated ptosis 
subsequent to descending paralysis. In a study, authors describe a patient with GBS who presented initially with isolated ptosis without ophthalmoplegia, and then he developed descending paralysis subsequently. ${ }^{21}$ Similarly, in our study, bilateral ptosis was observed in one patient as the first complaint which was followed by descending paralysis.

GBS presenting with only multiple cranial nerve dysfunction occurs very rarely. ${ }^{22}$ Pure cranial nerve involvement may occur, bilateral cranial neuropathy syndrome may develop without limb weakness. ${ }^{23}$ In our study group, one patient had multiple cranial nerve involvement, but the other physical and neurological manifestations were ordinary on first application.

Characteristic laboratory findings supporting GBS diagnosis are the absence of significant inflammatory findings (mononuclear cell count $<10 / \mathrm{mm}^{3}$ ), albuminocytological dissociation or disproportionate increase in CSF protein. ${ }^{5}$ Although protein levels may be normal during the first week of the illness, the majority of patients will experience an increase in protein levels if measured 2 or 3 weeks later. Previous studies have found that abnormal CSF protein elevation in GBS may be due to disorders that cause breakdowns in the blood-CSF barrier or the inflammatory reaction of the choroid plexus. $^{24}$ In our cases, CSF protein elevation was detected in 36 (80\%) cases and mean CSF protein was $80.6 \pm 58.5 \mathrm{mg} / \mathrm{dl}$. In our study, there was no significant difference in protein levels between AIDP and AMAN / AMSAN subtypes $(p=0.138)$.

Peripheral nerve demyelination in neurophysiological studies is manifested by slowing in nerve conduction. Both heavy demyelination and axonal damage cause a reduction in motor and sensory response amplitude. Most of the pediatric patients in the study by Pi-Lien et al. ${ }^{25}$ belonged to the AIDP group. The frequency of GBS in different clinical subtypes varies according to geographical area. The most common type of GBS in Europe is acute inflammatory demyelinating polyneuropathy (AIDP). It accounts for about $90 \%$ of all GBS patients. ${ }^{26}$ In a study in Japan, the proportion of children with AIDP (35\%) was similar to that of children with AMAN $(48 \%)$, and recovery was generally favorable in both subtypes. ${ }^{27}$ In our study axonal forms
(AMAN, AMSAN) and AIDP were found to be at approximate rate $(53.3 \%$ and $46.7 \%$, respectively). In the conducted studies, the AMAN pattern was found in $65-86 \%$ in China, $28,2930 \%$ in Argentina ${ }^{30}$ and $35 \%$ in Turkey. ${ }^{31}$ These findings suggest that the incidence of AMAN in childhood GBS varies considerably among countries. Although the etiology is unclear, preceding infectious agents and host factors are thought to be responsible. ${ }^{27}$

Nerve involvement in GBS may depend on the infectious agent. Campylobacter jejuni is commonly in association with AMAN, and anti-GMI and anti-GDIa IgG antibodies have been reported in $30 \%-40 \%$ of AMAN patients. ${ }^{32}$ Acute gastroenteritis caused by Campylobacter spp. is in the top three ranks along with Salmonella spp. and Shigella spp. in many parts of the world. The definitive diagnosis of Campylobacter infections is based on the microbiological examination and culture is the "gold standard" diagnostic method, but culture and isolation can be quite difficult. ${ }^{33}$ In our country, in 2012, it was observed that only $3.2 \%$ of clinical microbiology laboratories $(n=530)$ were able to isolate Campylobacter spp. according to the results of a study which was subjecting the current state of national microbiology laboratory capacity. ${ }^{34}$ In our patients, Campylobacter jejuni could not be isolated in stool cultures as well. Serologic test for Campylobacter jejuni and anti-ganglioside antibodies could not be performed in all patients for financial reasons. Ogawara et al. ${ }^{35}$ has reported the correlation between diarrhea secondary to Campylobacter jejuni and the GBS subtype AMAN. In our study, although Campylobacter jejuni could not be isolated, the history of gastroenteritis was significantly higher in patients with AMAN subtype $(\mathrm{p}=0.03)$.

The primary goal in the treatment of GBS is life support with intensive care treatment in the cases of respiratory insufficiency, prevention of secondary complications and intensive physical therapy, and the other goal is to try to stop the autoimmune process causing the demyelination of peripheral nerves by early immunomodulatory therapy. Immunomodulator treatments shorten the duration of the disease but do not significantly affect mortality. ${ }^{36}$ Intravenous immunoglobulin is generally 
preferred because of its ease of administration. ${ }^{6}$ Plasma exchange is a reliable and effective treatment for children over 10 kilograms. In studies conducted, plasmapheresis has been shown to be particularly useful when administered within the first seven days after the onset of symptoms. ${ }^{6}$ In a multicenter study involving mostly adults, it was shown that the efficacy of plasma exchange and intravenous immunoglobulin was similar and that the combination of both treatments was not superior to treatment alone. ${ }^{37}$

In our study IVIG was administered to 33 $(73.3 \%)$ patients, plasmapheresis and IVIG together was administered in nine (20\%), and plasmapheresis was administered to three $(6.7 \%)$ patients. Even though there are reports of increased risk of hypotension and spread of infection in plasmapheresis group; ${ }^{38}$ in our study, we did not observe any difference in terms of complications in different treatments.

The mean hospital stay was significantly longer in patients treated with combined plasma exchange and IVIG treatment $(p=0.001)$. This result may be due to our preference for plasma exchange and IVIG in patients with poor health. Especially, plasma exchange preferred mainly in the axonal forms with high GBS disability scores. In some previous studies, it is reported that plasma exchange is better than IVIG in children with GBS due to mechanical ventilation. ${ }^{39}$

GBS usually has a good prognosis, most patients completely recover with very slight motor loss. It has been reported that $62 \%$ to $92 \%$ of GBS patients develop fully functional healing in various studies..$^{8,10,40}$ In our study, $82.2 \%$ of the patients could walk without support at six months. Approximately 15$20 \%$ of patients require ventilation support, but respiratory failure does not persist. ${ }^{6,20}$ Autonomic dysfunction in pediatric patients is an important and independent risk factor for mechanical ventilation. ${ }^{41,42}$ In our study, six (13.3\%) patients required ventilation support. Autonomic dysfunction was seen in all of these patients, and bilateral facial paralysis was present in four patients (all of the bilateral facial paralysis patients). Although AIDP subtype has been reported in cranial nerve involvement, in our patients AMAN subtype was found more dominantly, and those patients had mechanical ventilation requirement.

Tekgül et al. ${ }^{31}$ have reported that during the first 12 months the recovery was slower in the AMAN group compared to the AIDP group, and after this duration, the recovery rates evened out. In our patients, GBS disability score at sixth months was significantly lower in AMAN subtype compared to AIDP subtype. In addition according to the GBS disability score, the mean time from onset of the disease to nadir, mechanical ventilation requirement, and presence of autonomic manifestation were found as poor prognostic factors.

Mortality rates vary between $3-13 \%$ in studies conducted so far. ${ }^{43}$ The most frequently described cause of death in GBS are respiratory failure. The estimated fatality rates of the cases which required mechanical ventilation for respiratory failure were $15-30 \% .{ }^{7}$ Autonomic instability is a potential initiating factor for fatal cardiac arrhythmias. Pulmonary infections have also been a cause of death during the recovery phase. ${ }^{43}$ Three of our cases $(6.7 \%)$ died due to unresponsive autonomic instability and respiratory infections.

Several studies have evaluated the factors that affect the prognosis in GBS, the reported factors include advanced age, premature infections, disease severity, axonal involvement, and mechanical ventilation, but these factors have not been confirmed with large prospective studies. ${ }^{1,10,24}$ In our study, the poor outcome on the sixth month was found to be associated with the short time interval between onset and admission, GBS disability score at nadir, AMAN subtype, the mean time from onset of illness to nadir, mechanical ventilation requirement, dysautonomia, and ventilator requirement.

GBS should be kept in mind in cases of acute ascending muscle weakness. Despite the fact that it is a self-limiting disease, the early detection and early treatment will reduce the length of stay in hospital, reduce morbidity and mortality. Although our cases had a greater degree of axonal form, full recovery was observed in the majority of patients. Plasma exchange and IVIG supportive care in selected cases should be the preferred treatment modality. In our study, the short duration of neurological deficit following infection, clinical stage of application, need for mechanical ventilation, dysautonomia, and 
current subtype were the negative prognostic factors. We concluded that dysautonomia and cranial nerve involvement which was associated with axonal involvement should be carefully monitored for ventilation needs.

\section{REFERENCES}

1. van Doorn PA, Ruts L, Jacobs BC. Clinical features, pathogenesis, and treatment of Guillain-Barré syndrome. Lancet Neurol 2008; 7: 939-950.

2. Visser LH, van der Meche FG, Meulstee J, et al. Cytomegalovirus infection and Guillain-Barre syndrome: The clinical, electrophysiologic, and prognostic features. Dutch Guillain-Barre Study Group. Neurology 1996; 47: 668-673.

3. Asbury AK. New concepts of Guillain-Barré syndrome. J Child Neurol 2000; 15: 183-191.

4. Susuki K, Koga M, Hirata K, Isogai E, Yuki N. A Guillain-Barré syndrome variant with prominent facial diplegia. J Neurol 2009; 256: 1899-1905.

5. Asbury AK, Cornblath DR. Assessment of current diagnostic criteria for Guillain-Barre syndrome. Ann Neurol 1990; 27(Suppl): S21-S24.

6. Ryan MM. Guillain-Barre Syndrome in childhood. J Paediatr Child Health 2005; 41: 237-241.

7. Hung PL, Chang WN, Huang LT, et al. A clinical and electrophysiologic survey of childhood Guillain-Barré syndrome. Pediatr Neurol. 2004; 30: 86-91.

8. Soysal A, Aysal F, Caliskan B, et al. Clinicoelectrophysiological findings and prognosis of GuillainBarre' syndrome 10 years experience. Acta. Neurol Scand 2011; 123: 181-186.

9. Gupta D, Nair M, Baheti NN, Sarma PS, Kuruvilla A; Diplomate-American Board.Electrodiagnostic and clinical aspects of Guillain-Barré syndrome: An analysis of 142 cases. J Clin Neuromuscul Dis 2008; 10:42-51.

10. van Koningsveld R, Steyerberg EW, Hughes RAC, Swan AV, van Doorn PA, Jacobs BC. A clinical prognostic scoring system of Guillain-Barré syndrome. Lancet Neurol 2007; 6: 589-594.

11. Rantala H, Uhari M, Niemela M. Occurence, clinical manifestations, and prognosis of Guillain-Barre Syndrome. Arch Dis Child 1991; 66: 706-708.

12. Rees JH, Thompson RO, Smeeton NC, Hughes RAC. Epidemiological study of Guillain-Barre Syndrome in south cast England. J Neurol Neurosurg Psychiatry 1998; 64: 74-77.

13. Emilia-Romagna Study Group on Clinical and Epidemiological Problems in Neurology. Guillain Barre syndrome variants in Emilio-Romagna, ltaly, 1992-3: incidence, clinical features and prognosis. J Neurol Neurosurg Psychiatry 1998; 65: 218-224.

14. Delanoe C, Sebire G, Landrieu P, Huault G, Metral S. Acute inflammatory demyelinating polyradiculopathy in children: Clinical and electrodiagnostic studies. Ann Neurol 1998; 44: 350-356.
15. Rantala H, Uhari M, Cherry JD, Shields WD. Risk factors of respiratory failure in children with GuillainBarré syndrome. Pediatr Neurol 1995; 13: 289-292.

16. Hicks CW, Kay B, Worley SE, Moodley M. A clinical picture of Guillain-Barré syndrome in children in the United States. J Child Neurol 2010; 25: 1504-1510.

17. Cuadrado JI, de Pedro Cuesta J, Ara JR, et al; Spanish GBS Epidemiological Study Group. Guillain- Barré syndrome in Spain, 1985-1997: epidemiological and public health view. Eur Neurol 2001; 46: 83-91.

18. Gensicke H, Datta AN, Dill P, Schindler C, Fischer D. Increased incidence of Guillain-Barre syndrome after surgery. Eur J Neurol 2012; 19: 1239-1244.

19. Haber P, Sejvar J, Mikaeloff Y, DeStefano F. Vaccines and Guillain-Barré syndrome. Drug Saf 2009; 32: 309323.

20. Bradshaw DY, Jones HR Jr. Guillain-Barre syndrome in children: Clinical course, electrodiagnosis, and prognosis. Muscle Nerve 1992; 15: 500-506.

21. Teng HW, Sung JY. Ptosis as the initial presentation of Guillain-Barré syndrome. J Emerg Med 2012; 43 e283-e285.

22. Munsat TL, Barnes JE. Relation of multiple cranial nerve dysfunction to the Guillain-Barré syndrome. J Neurol Neurosurg Psychiatry 1965; 28: 115-120.

23. Yu JY, Jung HY, Kim CH, Kim HS, Kim MO. Multiple cranial neuropathies without limb involvements: Guillain-Barre syndrome variant? Ann Rehabil Med 2013; 37: 740-744.

24. Okuyama $\mathrm{H}$. Mechanism of increase of the cerebrospinal fluid (CSF) protein content in Guillain-Barre syndrome. Study using RISA in evaluation of diffusion within CSF cavity and transport of CSF to circulating plasms. Rinsho Shinkeigaku (Clin Neurol) 1975; 15: 817-826.

25. Hung PL, Chang WN, Huang LT, et al. A clinical and electrophysiologic survey of childhood Guillain-Barre syndrome. Pediatr Neurol 2004; 30: 86-91.

26. Vucic S, Kiernan MC, Cornblath DR. Guillain-Barre syndrome: an update. J Clin Neurosci 2009; 16: 733741.

27. Nagasawa K, Kuwabara S, Misawa S, et al. Electrophysiological subtypes and prognosis of childhood Guillain-Barre syndrome in Japan. Muscle Nerve 2006; 33: 766-770.

28. Lu JL, Sheikh KA, Wu HS, et al. Physiologic-pathologic correlation in Guillain-Barre syndrome in children. Neurology 2000; 54: 33-39.

29. McKhann GM, Cornblath DR, Griffin JW, et al. Acute motor axonal neuropathy: A frequent cause of acute flaccid paralysis in China. Ann Neurol 1993; 33: 333342.

30. Paradiso G, Tripoli J, Galicchio S, Fejerman N. Epidemiological, clinical, and electrodiagnostic findings in childhood Guillain-Barre syndrome: A reappraisal. Ann Neurol 1999; 46: 701-707.

31. Tekgul H, Serdaroglu G, Tutuncuoglu S. Outcome of axonal and demyelinating forms of Guillain-Barre syndrome in children. Pediatr Neurol 2003; 28: 295299. 
32. Kalita J, Misra UK, Goyal G, Das M. Guillain-Barré syndrome: subtypes and predictors of outcome from India. J Peripher Nerv Syst 2014; 19: 36-43.

33. Jerris RC, Fields PI, Nicholson MA. Fecal culture for Campylobacter and related organisms. In: Garcia LS, Isenberg HD (eds). Clinical Microbiology Prosedures Handbook. (2nd ed), ASM Press, Washington D.C. 2010:3.8.2.1 - 3.8.2.16.

34. T.C. Sağlık Bakanlığı (Bulaşıcı Hastalıkların Sürveyansı ve Kontrolü Projesi TR0802.16-01 Avrupa Birliği ve Dünya Bankası desteği ile) (Akbaş E, Pr Danışmanı). Türkiye'de Bulaşıı Hastalıkların Tanısında Mikrobiyoloji Laboratuvar Kapasitesi Mevcut Durum Değerlendirmesi: Anket - LabKap2012. XXXV. Türk Mikrobiyoloji Kongresi, Kuşadası, 4 Kasım 2012.

35. Ogawara K, Kuwabara S, Mori M, Hattori T, Koga M, Yuki N. Axonal Guillain-Barré syndrome: Relation to anti-ganglioside antibodies and Campylobacter jejuni infection in Japan. Ann Neurol 2000; 48: 624-631.

36. Haupt WF. Recent advances of therapeutic apheresis in Guillain-Barre syndrome. Ther Apher 2000; 4: 271-274.

37. Plasma Exchange/Sandoglobulin Guillain-Barre syndrome Trial Group. Randomized trial of plasma exchange, intravenous immunoglobulin, and combined treatments in Guillain-Barre syndrome. Lancet 1997; 349: $225-230$.
38. Chen WH, Yeh JH, Chiu HC. Experience of double filtration plasmapheresis in the treatment of GuillainBarré syndrome. J Clin Apher 1999; 14: 126-129.

39. El-Bayoumi MA, El-Refaey AM, Abdelkader AM, ElAssmy MM, Alwakeel AA, El-Tahan HM. Comparison of intravenous immunoglobulin and plasma exchange in treatment of mechanically ventilated children with Guillain Barré syndrome: a randomized study. Crit Care 2011; 15: R164.

40. Korinthenberg R, Schessl J, Kirschner J. Clinical presentation and course of childhood GuillainBarré syndrome: a prospective multicentre study. Neuropediatrics 2007; 38: 10-17.

41. Dimario FJ Jr, Edwards C. Autonomic dysfunction in childhood Guillain-Barré syndrome. J Child Neurol 2012; 27: 581-586.

42. Samadi M, Kazemi B, Golzari Oskoui S, Barzegar M Assessment of autonomic dysfunction in childhood guillain-barré syndrome. J Cardiovasc Thorac Res 2013; 5: 81-85.

43. van den Berg B, Bunschoten C, van Doorn PA, Jacobs BC. Mortality in Guillain-Barre syndrome. Neurology 2013; 80: 1650-1654. 